\title{
Visualizing student engagement in e-learning environment
}

\author{
Enrica Pesare, Teresa Roselli, Veronica Rossano \\ Dipartimento di Informatica \\ Università degli Studi di Bari Aldo Moro \\ Bari, Italy \\ \{enrica.pesare, teresa.roselli. veronica.rossano\}@uniba.it
}

\begin{abstract}
The learning assessment in e-learning contexts is one of the latest challenges for educational technology researchers. One of the main issues to be addressed is the definition of dimensions that should be used to measure the learning effectiveness. In this perspective, the research work aims at defining the engagement indicators useful to assess the active participation of students in social learning environments. Moreover, the paper presents the design and implementation of Learning Dashboards aimed at visualizing the student engagement in online communities where the engagement and involvement of students are the key factors for successful learning.
\end{abstract}

Keywords- Assessment; Learning Analytics; Learning dashboard; Social learning environments; Engagement.

\section{INTRODUCTION}

The learning assessment is one of the main issues in the educational field. In the latest years, the interest in this topic is rising thanks to the evolution of e-learning methods and techniques. The new teaching models adopted in the MOOCs (Massive Open Online Courses) [1] [2] require the adoption of new tools and measures that allow teachers to make effective and reliable assessment even with a large population of learners. In the literature, different solutions have been proposed, such as the adoption of qualitative measures [3] [4] to have detailed information about the interaction of the learner with the peers and the didactic resources, or the definition of tools able to supply detailed information on the student learning path, starting from the tracking data of e-learning platform [5] [6]. For these reasons, the interest of the educational technology researchers is focusing on Learning Dashboards that allow, both faculty and students, to tune their actions (lectures, assignments, study) based on rapid feedbacks on student progress in learning [7]. Currently, the interactions are becoming even more complex in the e-learning environments. Thus, the number of dimensions to take into account to make a successful assessment of the student's learning path is improving. The main challenge of Educational Technology field is to point out all the dimensions useful to measure the effectiveness of the e-learning paths [8].

DOI reference number: 10.18293/DMS2016-028
In this perspective, our point of view is that in the elearning environments 2.0, where the construction of the knowledge is shared among the different users, to measure the engagement in the e-learning process could give information about the learning effectiveness. For this reason, the paper presents a Learning Dashboard aimed at visualizing the student engagement in web based learning environment. In particular, the attention is focused on online communities, where the engagement and involvement of students is the key factor for a successful learning.

\section{RELATED WORKS}

The Learning Dashboards are commonly used in a wide range of e-learning environments: Learning Management Systems, Web-based and Personal Learning Environments, Massive Open Online Courses and so on. Information Visualization techniques, indeed, are powerful tools in the learning analytics research field, since they allow to visualize the collected data about student's activities. Visualization can impact on the user behavior and motivation, for both students and teachers, and promote self-awareness and reflection about the learning process [9].

The interest in this research field and the number of proposed approaches are growing in the latest years. For ours research goals, we decided to narrow the research on two important aspects in e-learning environments: student's progresses and performances and student's engagement.

\section{A. Visualizing progresses and performaces in e-learning enviroment}

Numerous are the solutions proposed to face this issue. Student Activity Meter (SAM) [10] [11], for example, was designed to explore the classroom activities. It provides an overview of student's activities through simple statistics. For each student, the indicators are the time spent and the documents used. These indicators are compared with minimum, maximum and average values of the whole class.

The first indicator, time spent, is displayed with a line chart visualization; it shows a line for every student in the course; the horizontal axis shows the dates and the vertical axis displays the total time spent [11]. Indeed the visualization of 
activities over time is a key aspect when analyzing student behaviors. The second indicator, document used, is not directly displayed but it is used to provide recommendations with a simple recommender system.

Furthermore a parallel coordinates chart shows correlations among (i) the total time spent on the course, (ii) the average time spent on a document, (iii) the number of documents used and (iv) the average time of the day that the students work [11] allowing the discovery of patterns in the student's behavior.

Mastery Grids introduces the so called "social progress visualization" [12] to engage students and to guide them through the learning resources exploration. The indicators used are the progress made by each student. The progresses are presented in a matrix where for each domain topic (horizontal dimension) and kind of resources available for that topic (vertical dimension) the level of completeness or level of progress a learner has for the given combination of topic and resource type is showed through color intensity (third dimension).

In addition to the individual progress visualization, Mastery Grids enables comparison with other peers (the class average, the top ranking students) and highlights the differences between the individual user and the group. Furthermore, it enables more compact and detailed comparison selecting one kind of resource. This direct comparison pushes the user to improve her/his activity by stimulating them to complete different kind of activities and to access new content at the same time.

CAM Dashboard was designed to allow the exploration of learners' behavior in Personal Learning Environments and to enable both self-reflection and comparison with peers to improve student's motivation [13] [14]. It collects data from different data sources even outside the traditional LMS, and provides visualization according to the student's goals.

The indicators are the total number of activities done by the student and the number of events or time spent for each application used by the student over time. Furthermore those indicators are grouped by day of the week, by action performed or by resources involved. Noteworthy is the distinction of different kind of actions that enables a deeper level of analysis, for instance the distinction of active and passive actions such as writing and reading activities.

The main visualization is a line chart of the activity over time (annotated timeline) while the grouped indicators are presented with bar chart visualizations that allow the comparison between the time spent by the user and the average time of peers.

VeeU was designed to enhance student assessment in distance learning environment for both teachers and students. [5]. The indicators proposed are the number of daily accesses, the number and distribution of activities in a course and the completion rate of course activities.

The accesses are presented in a time line visualization at different aggregation levels (different courses or single course) for the teacher, while the student visualizes the number of her/his daily accesses compared to the average values for the class. A pie-chart visualization is used for the percentage and distribution of activities while the completion rate is displayed in a gauge chart visualization with a list of recommendations to help student in achieving goals.

Even StepUp! has been designed for students empowerment in open learning environments [15][16][17] in the "quantified self" perspective [18]. It collects tracking data from group blogs and twitter (post, comments, tweet). Even in this case the indicators are the number of activities and the time spent but the activities are also classified in assimilative (blogging and writing reports), communicative (twitter and comments) and productive activities (programming) [19] enabling distinction of active and passive behavior in this case too.

The indicators are presented with numerical data and sparklines for every student to provide a quick overview. The sparklines can be detailed in a stacked bar chart visualization that displays activity over the weeks, grouped by kind of activities and participation to promote awareness of what students did and how they spent their time.

TrAVIS [20] collects data about communication activities in distance learning environments to promote self-monitoring. It distinguishes four levels of interaction (aggregation, discussion, cooperation and collaboration) that correspond to four levels of indicators:

- aggregation level: connection frequency, threads started, messages posted, messages replied, and messages quoted. These indicators are commonly used to describe the activities of each individual student;

- discussion level: browsing, forums, posting, reading and chatting activities. These indicators are useful to estimate the user interest in the discussions or her/his level of community interaction;

- cooperation level: thread started, new messages, replied messages, quoted messages, files uploaded, files downloaded and participation level. These indicators are useful to evaluate the contribution of each user to the community.

- collaboration level: even these indicators are constructed from the lower levels indicator but in this case the focus is moved from the individual perspective to the group level perspective. As a matter of fact they are collected at group level within a defined timespan in order to compare the participation rates or the productivity rates of different groups.

The visualization technique at each level is the spider chart: a spider chart for each user (at the aggregation, discussion and cooperation levels) or for each group (at the collaboration level) allows visual comparison among students and among groups. 


\section{B. Visualizing engagement in e-learning enviroments}

In the e-learning field, the increasing interest in the engagement dimension has led to the definition of different dashboards to monitor and improve student engagement.

VisEN, for example, is addressed to students to allow the exploration of data about engagement in form of visual narratives [21] [22]. It presents the student's engagement in a quick gauge chart while further details are provided on demand. Students can interact with visualizations to realize how engagement score was calculated, based on course interaction (page click), study time (reading and reviewing durations), submissions and questionnaire scores. They can compare their engagement with peers (all class members or similar engaging peers) at different levels (global score or activity level).

Even in the emerging field of MOOCs, student engagement has acquired a great importance. Coffrin [23] proposes the analysis of student's activities in MOOCs using visualization techniques. In this case, the visualizations are used at macro level to understand patterns of student engagement. However, even if the proposed analysis are not strictly related to our goal, they give us some interesting points of view on the topic. The analysis starts from two simple histograms of students' participation and assessment performance, broken down by week. This first visualization confirms the common pattern of high interest at the beginning with increasing attrition rate over time, but breaking down the data, a deeper understanding of engagement is provided. In particular, students in a MOOC can be classified into Auditors, Active and Qualified students according to the kind of activities performed (video lectures and assessments). This breakdown can be useful in different visualization (histogram or state transition diagram) to closely analyze the percentage and relative proportion of students and to understand the temporal evolution of engagement trajectories [24].

Apart from individual participation in MOOC, even the social interactions have a great influence in particular when involving social learning processes [25].

Schreurs [26] proposes the application of Social Learning Analytics in a MOOC platform. In particular, it focuses on the network visualization from discussion forums: every user is a node, every reply to a post is a tie, while a simple tag cloud allows the filtering of ties and nodes based on the contents. Moreover a tooltip will allow the discovery of topic of interest and expertise of every user. Then different network perspectives can promote reflection on learners' interaction or make visible the contents developed through discussions.

Another perspective on engagement in web-based learning environment is given in [27] where the authors propose the visual representation of cognitive and behavioral indicators of engagement to support teachers in monitoring learners' engagement. To reflect the multidimensionality of engagement the indicators are built from student participation actions (behavioral indicators) and from modification actions on the learning documents structures (cognitive indicators). The first ones are computed from number of actions and duration (number of login, number accesses to a learning resource, time spent on a resource etc.) while the latter are computed from a particular subset of action related to the structural modification of learning documents (create, add, update, delete, move and insert).

The proposed visualization is based on the small multiple visualization technique for each student's session: each frame represents a simplified mind map at different times $\left(t_{i}\right)$ with dotted lines for deleted elements and solid lines for existing ones. The small multiple representation can be combined with a linear representation for each node where the structural modifications on the selected node are made visible over time. The small multiple visualization might have problems in case of growing numbers of nodes or growing number of time intervals while the linear representation is easily manageable for temporal data even if it does not allow comparisons of data about different nodes at the same time.

\section{VEEU 2.0}

The main goal of our research is to make students and teachers aware of their engagement in a social learning environment. As a matter of fact, teachers and students need to be aware of what kinds of interactions are occurring in the virtual space and how the building up knowledge process happens. This is the so called "situational awareness" that, according to Few's principle design [28], is one of the main purposes of dashboards. In this perspective, the rapid perception of information through the dashboard is fundamental to facilitate the decision-making process.

To achieve these goals, in the following sections are briefly presented the social learning environment and the dashboard design process, from the data analysis (to define the most suitable predictors and indicators), to the selection of the best visualization techniques (to depict the relevant data at a glance).

\section{A. Social Learning Environment}

The learning environment, as depicted in Fig. 1, is a customized Moodle instance, in order to preserve consistency with the learning management system in use in our University. To enable Moodle to adopt the social paradigm the SocialWall plug-in, (https://moodle.org/plugins/format_socialwall) was used. It turns the traditional Moodle course format into a social interface. Moreover, a Wiki activity as collaborative knowledge repository has been added. In particular, the Wiki enables users to co-create a complex web document, even without any knowledge about HTML, allowing them to gather pieces of knowledge and build a shared repository.

The SocialWall enables users to post messages, documents, links and any other kind of resources already available in Moodle. Users can express their like or comment the posts. All the social activities will appear on a timeline provided with some filtering tools. 


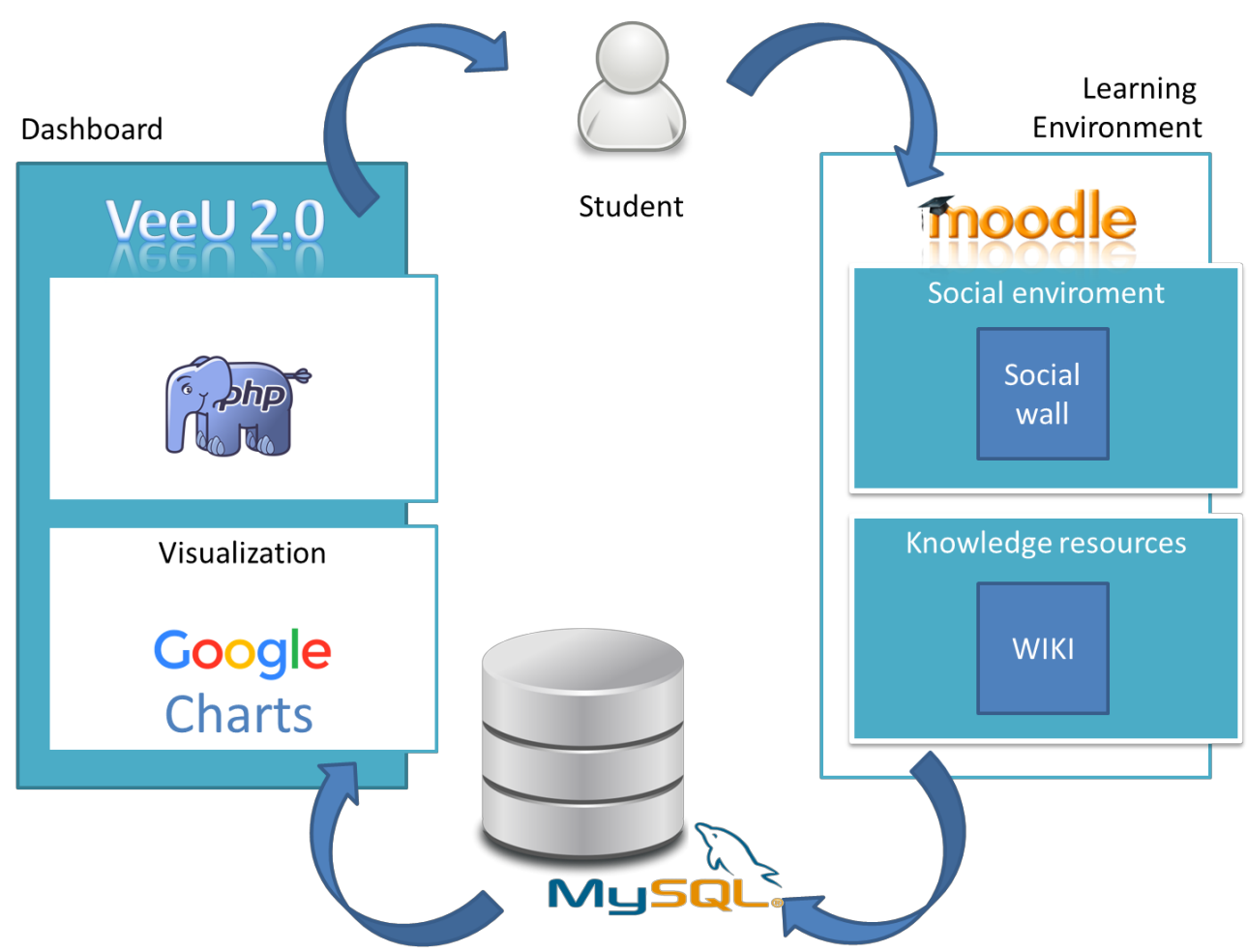

Figure 1. The Social Learning Environment

All the interactions occurring in the social learning environment are traced and stored in the Moodle database that is the data source of our dashboard. The trace-based approache, indeed, is one of the best method used in literature to detect users engagement, since they gather a lot of information without interfering with the learning activities [29] [30].

\section{B. Engagement indicators}

Engagement has been deeply studied in technology enhanced learning research from a wide range of perspectives: it is commonly recognized as a multidimensional and multifaceted construct but the definition of engagement is context dependent. In the specific context of social learning environments the engagement is strictly related to the activities performed in the community and to the users' participation in different kinds of activities.

First of all, it is necessary to classify the level of user's participation in the community, from a lower level, or peripheral participation [31], mainly consisting of reading resources and limited interactions with other users, to a higher level, or more active participation, consisting of activities such as writing comments on other users posts, sharing knowledge resources enabling a more responsible contribution to the knowledge of the community.
According to this perspective, in our research the engagement indicators were defined as follow:

- Passive interactions

- Number of likes received by a post or a comment

- Number of wiki pages read

- Active interactions

- Number of posts published on the social wall

- Number of comments shared on the social wall

- Numbers of created wiki pages

- Number of revised wiki pages

Furthermore, the next step is to identify the subject of interest of the user's participation. As defined in [32] the indicators of engagement are based on the participation both in social life of the community (interaction with other participants) and in the knowledge building activities (interaction with the knowledge resources).

According to the course structure we defined the engagement indicators as follow: 
- Social Interactions

- Number of posts published on the social wall

- Number of comments shared on the social wall

- Number of likes received on a post or a comment

- Knowledge Interactions
- Number of wiki pages read
- Number of created wiki pages
- Number of revised wiki pages

All the indicators are calculated from the traces collected in the Moodle database: in particular standard Moodle log tables has been used for the indicators based on the Wiki while the SocialWall log tables have been used for the related indicators.

The basic indicators are collected at daily intervals and are then aggregated weekly or monthly according to the required level of analysis. The common aggregation level makes the indicators comparable over time and allows the timely evolution of engagement to be assessed. Then, as depicted in Fig. 1, the calculated indicators are presented through Google Chars using the most appropriate visualization as detailed in the next section.

\section{Visualization}

Our first goal was to visualize information about the entire class in order to understand the behavior and the level of participation of each student. Then we use our first pair of indicators to display the position of each student on a scatterplot. The students' position in the two-dimensional area is defined according to the number of Passive Interactions in a week on the $\mathrm{x}$-axis and the number of Active Interactions in a week on the y-axis (Fig. 2). This visualization will provide a quick overview of the current status of the entire class, thanks to the scatterplot ability to display trends and relationship in a cloud of points [33]. Furthermore, outlier students will be easily identified but, in order to understand the reason of their poor participation, a detailed visualization is required.

To analyze the behavior of single student a visualization of engagement trends over time is needed. Thus, the second visualization (Fig. 3) depicts the details of a particular student in terms of trends and distribution. It is based on a linear visualization, a time series: two lines (one for each indicator) will display the trends of the engagement indicators over time for Passive and Active Interactions. In this case the indicators can be collected using daily, weekly or monthly intervals according to the required level of analysis.

Furthermore a pie chart will display the distribution of interaction in a selected time interval, between passive and active interactions, but also among the different components of each indicator (Number of likes received, Number of wiki pages read vs Number of posts published, Number of comments shared, Numbers of created wiki pages and Numbers of revised wiki pages).

After the analysis of the level of participation, the attention was focused on the kind of engagement. As a matter of fact, the more passive or active attitude of a student is not enough to evaluate the students' engagement.

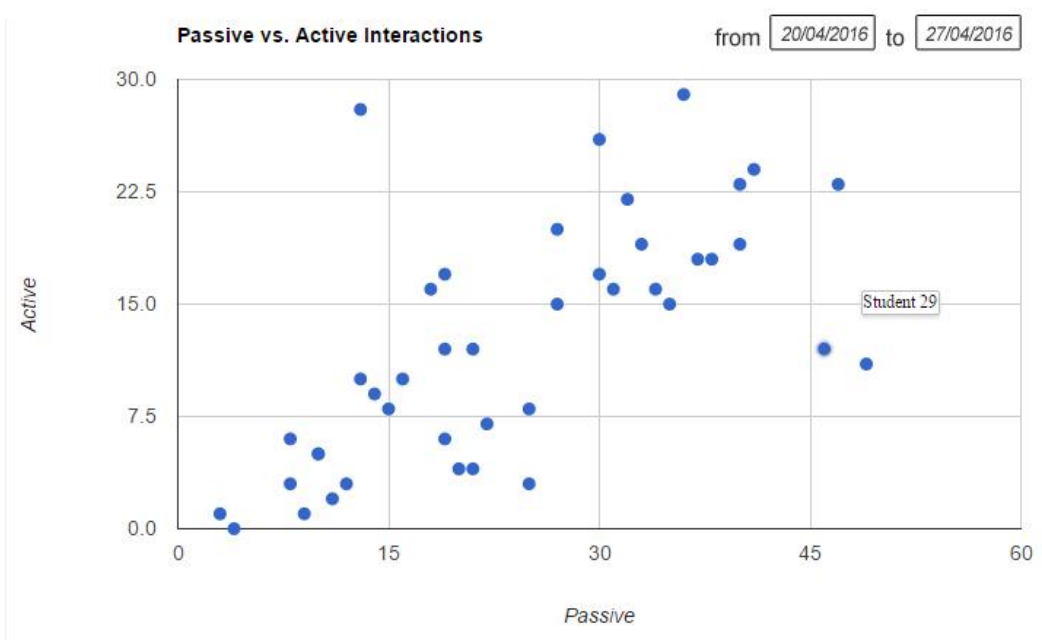

Figure 2. First visualization:

Scatterplot of Passive vs Active Interactions 

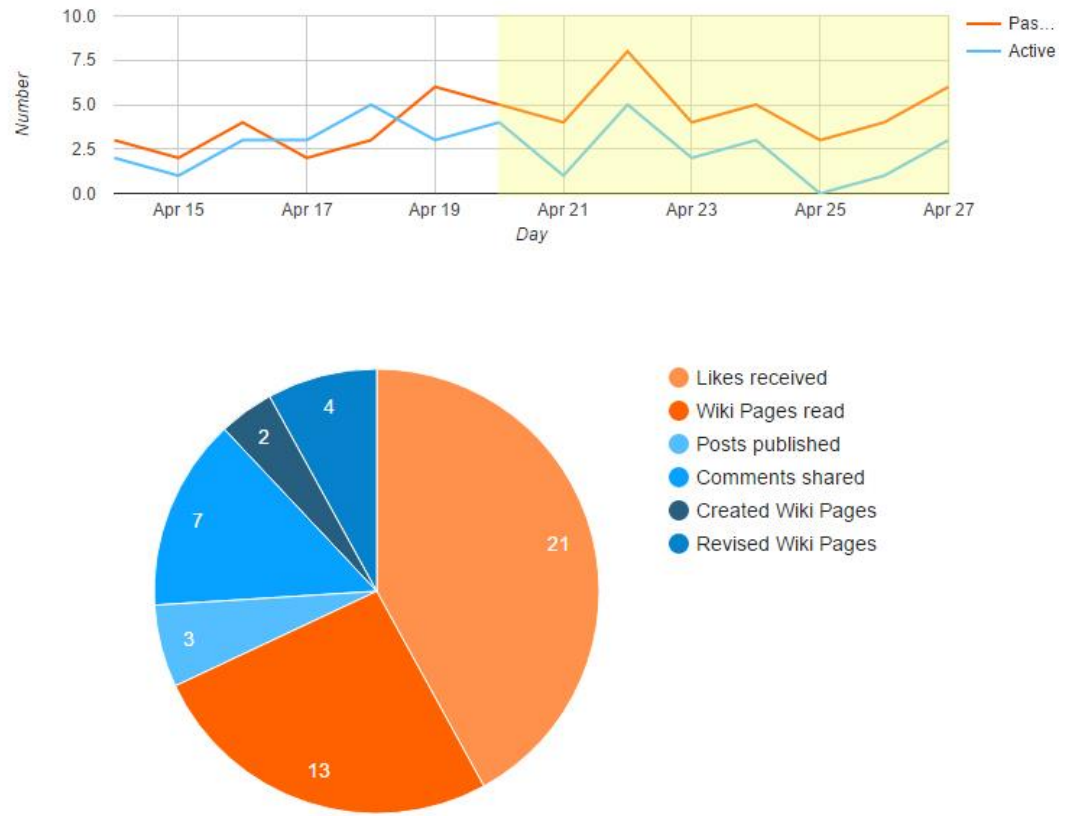

Figure 3. Second Visualization:

Trends and distribution of Passive vs Active Interactions for a single student

The third visualization (Fig. 4) is a scatterplot that, in this case, displays the students' position according to the number of Social Interactions in a week on the x-axis and Knowledge Interactions in a week on the $y$-axis. This kind of visualization will provide a quick overview about the subject of interests of student's engagement allowing the identification of purely socializer users or users actually involved in both Social and Knowledge Interactions.
A detailed visualization of a particular student will be useful in this case too. The fourth visualization (Fig. 5) presents the details of a particular student in term of trends and distribution with a time line and a pie chart built with this new pair of indicators.

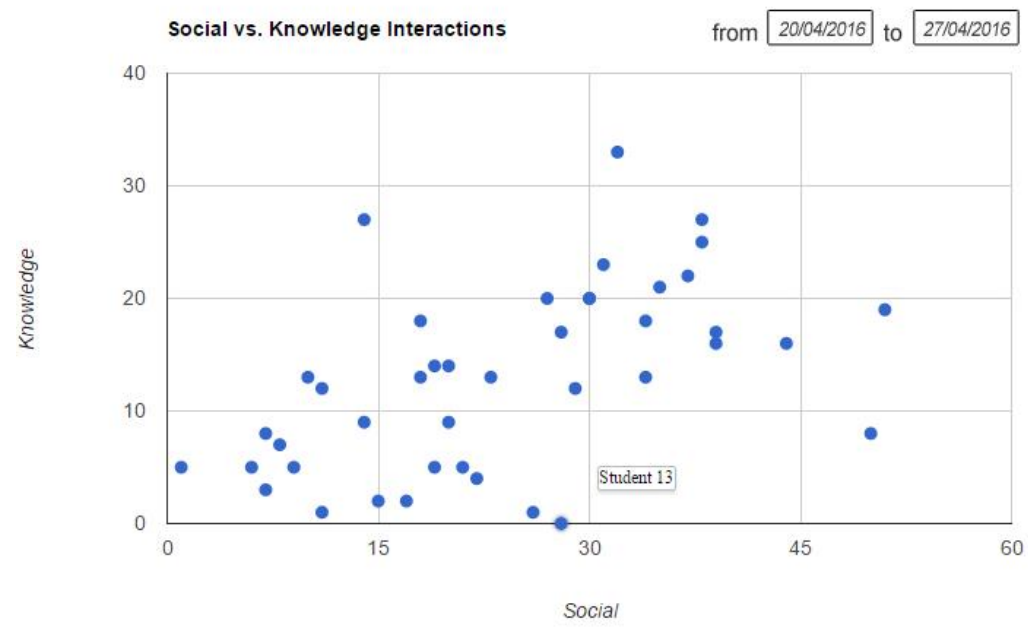

Figure 4. Third visualization:

Scatterplot of Social vs Knowledge Interactions 

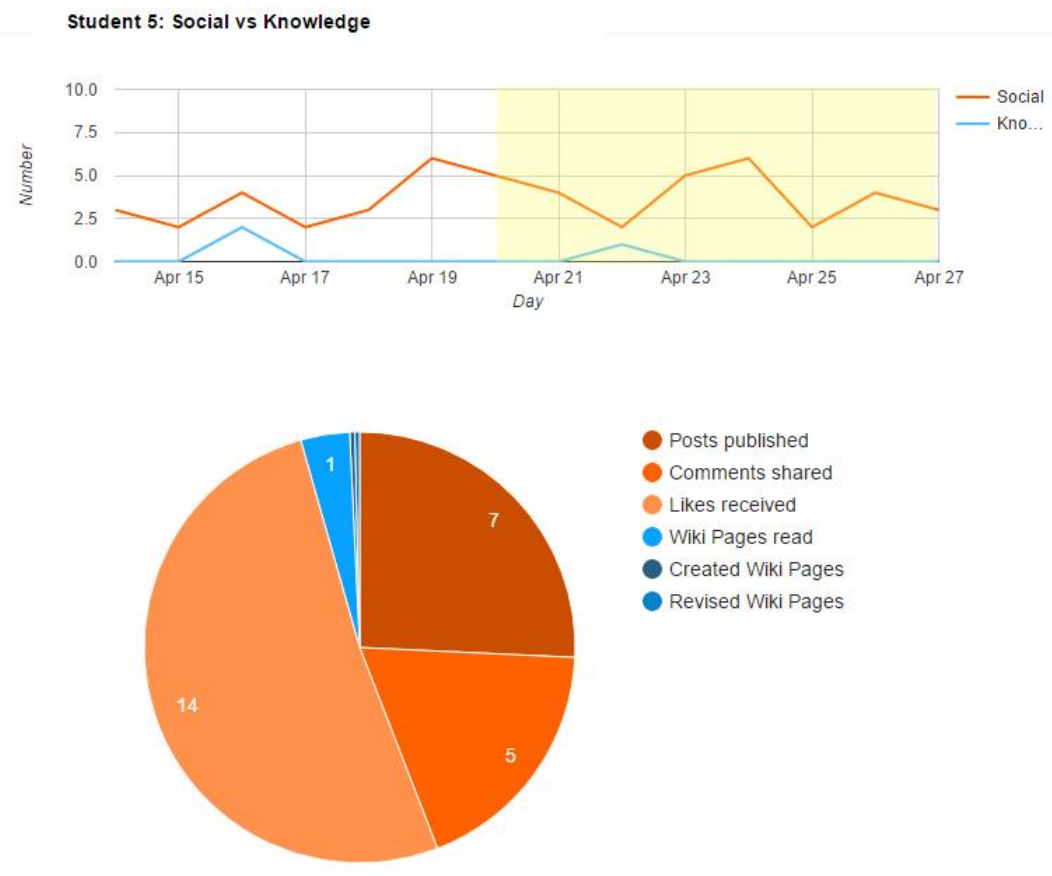

Figure 5. Fouth Visualization:

Trends and distribution of Social vs Knowledge Interactions for a single student

\section{CONCLUSION AND FUTURE WORKS}

Our research aims to address the needs of information about the learning effectiveness in social learning environment, using engagement measures.

To this end, activity monitoring tools have become vital in distance education, thanks to the increasing availability of data collected by tracking the online activities. Information Visualization techniques, taking advantage of visual perception skills, are powerful tools to present a large amount of data by transforming traces in information. An accurate dashboard design is essential to provide students and teachers with a decision support tool that (1) promotes the awareness of what is happening in the learning process, (2) encourages personal reflection and achieving goals.

Data and reports usually provided by learning management systems are usually limited to measurement of activities and performances but there is a growing need to monitor less tangible aspects such as engagement in the social learning contexts. This is the main aim of the proposed dashboards, which is still under development. The approach adopted is an iterative process, which involves both researchers and social learning environment users. Researchers in the first design phase have involved teachers in order to define the most relevant indicators and to define the most appropriate visualization for the dashboards. The student were engaged in the pilot study, which involved two classes of undergraduate students from the Computer Lab courses held by the Department of Education, Psychology and Communication of the University of Bari Aldo Moro. Students are currently working in the social learning environment and the results of the beta test phase of the dashboard will be soon available. First results seem promising but further field test are required for both to measure the long-term effectiveness of dashboards in the learning process and to improve dashboard functionalities.

Future developments are under investigation. In particular, we are interested in monitoring the evolution of engagement indicators over time both for each single student and for the whole class. Static or dynamic time mapping approaches will be evaluated. Static visualization, such as small multiples, is more effective for analysis, whereas dynamic visualization, such as animation, was found to be more effective for presentation [34]. As stated by Chevalier [35], animations are commonly used (1) to replay history of events that occurred in a dynamic system allowing users to go back in time, and (2) to make activities and change visible at the same time, while keeping the user engaged thanks to the ability to attract user's attention, However, animation is not immune to fault such as higher cognitive load or perceptual effects of change blindness [36]. In order to overcome this drawbacks interactivity may be the key to overcome the difficulties of perception and comprehension [37] allowing users to explore time dimension, if needed, without distracting them from focusing on a single moment in time.

Furthermore, other indicators will be required to provide users with further insight of what is happening in the social learning environment. In particular, Social Network Analysis measures and social network visualization will allow us to 
make explicit the relationships between users; Discourse and Content Analysis will allow us to explicit the topic of interest [38]. Even in this case interactive visualization will be useful to analyze and present the available data: for instance a tag-cloud visualization of topics will allow users to refine engagements indicator based on a most relevant or on a specific topic.

\section{REFERENCES}

[1] J. Kennedy, "Characteristics of Massive Open Online Courses (MOOCs): A Research Review, 2009-2012.,” J. Interact. Online Learn., vol. 13 , no. $1,2014$.

[2] T. R. Liyanagunawardena, A. A. Adams, S. A. Williams, and T. Rekha Liyanagunawardena, "MOOCs: a systematic study of the published literature 2008- 2012," Int. Rev. Res. Open Distance Learn., vol. 14, no. 3, pp. 202-227, 2013

[3] W. Admiraal, B. Huisman, and O. Pilli, "Assessment in massive open online courses," Electron. J. e-Learning, vol. 13, no. 4, pp. 207-216, 2015.

[4] P. J. Muñoz-Merino, J. a. Ruipérez-Valiente, C. Alario-Hoyos, M. Pérez-Sanagustín, and C. Delgado Kloos, "Precise Effectiveness Strategy for analyzing the effectiveness of students with educational resources and activities in MOOCs," Comput. Human Behav., vol. 47, pp. 108-118, 2015.

[5] E. Pesare, T. Roselli, V. Rossano, and P. Di Bitonto, "Digitally enhanced assessment in virtual learning environments," J. Vis. Lang. Comput., vol. 31, pp. 252-259, 2015.

[6] G. Siemens, "Learning Analytics: Envisioning a Research Discipline and a Domain of Practice," 2nd Int. Conf. Learn. Anal. Knowl., no. May, pp. 4-8, 2012.

[7] D. Bajzek, W. Brown, M. Lovett, and G. Rule, "Inventing the digital dashboard for learning," World Conf. Educ. Multimedia, Hypermedia Telecommun. 2007, pp. 1084-1092, 2007.

[8] G. Hwang, "Definition, framework and research issues of smart learning environments - a context-aware ubiquitous learning perspective," Smart Learn. Environ., vol. 1, pp. 1-14, 2014.

[9] K. Verbert, S. Govaerts, E. Duval, J. L. Santos, F. Van Assche, G. Parra, and J. Klerkx, "Learning dashboards: An overview and future research opportunities," Pers. Ubiquitous Comput., vol. 18, no. 6, pp. 1499-1514, 2014.

[10] S. Govaerts, K. Verbert, E. Duval, and A. Pardo, "The student activity meter for awareness and self-reflection,” Chi, pp. 869-884, 2012.

[11] S. Govaerts, K. Verbert, J. Klerkx, and E. Duval, "Visualizing activities for self-reflection and awareness," Lect. Notes Comput. Sci. (including Subser. Lect. Notes Artif. Intell. Lect. Notes Bioinformatics), vol. 6483 LNCS, pp. 91-100, 2010.

[12] T. D. Loboda, J. Guerra, R. Hosseini, and P. Brusilovsky, "Mastery Grids : An Open Source Social Educational Progress Visualization," pp. 235248, 2014.

[13] J. L. Santos, K. Verbert, S. Govaerts, and E. Duval, "Visualizing PLE usage," CEUR Workshop Proc., vol. 773, pp. 34-38, 2011.

[14] J. L. Santos, S. Govaerts, K. Verbert, and E. Duval, "Goal-oriented visualizations of activity tracking: a case study with engineering students," Learn. Anal. Knowl., pp. 143-152, 2012.

[15] J. L. Santos, K. Verbert, and E. Duval, "Empowering students to reflect on their activity with stepup!: Two case studies with engineering students.," CEUR Workshop Proc., vol. 931, pp. 73-86, 2012.

[16] J. L. Santos, K. Verbert, S. Govaerts, and E. Duval, "Addressing learner issues with StepUp!,” Proc. Third Int. Conf. Learn. Anal. Knowl. $L A K ' 13$, p. 14, 2013.

[17] K. Verbert, E. Duval, J. Klerkx, S. Govaerts, and J. L. Santos, "Learning Analytics Dashboard Applications," Am. Behav. Sci., no. February, p. $0002764213479363-, 2013$.
[18] V. Rivera-Pelayo, V. Zacharias, L. Müller, and S. Braun, "Applying quantified self approaches to support reflective learning," Proc. 2nd Int. Conf. Learn. Anal. Knowl. - LAK '12, p. 111, 2012.

[19] G. Conole and K. Fill, "A Learning Design Toolkit to Create Pedagogically Effective Learning Activities,” J. Interact. Media Educ., vol. 8, pp. 1-16, 2005.

[20] M. May, S. George, and P. Prévôt, "TrAVis to Enhance Students' Self-monitoring in Online Learning Supported by Computer-Mediated Communication Tools," Ijcisim, vol. 3, no. May 2016, pp. 623-634, 2011.

[21] B. Yousuf and O. Conlan, "Enhancing Learner Engagement through Personalized Visual Narratives," 2014 IEEE 14th Int. Conf. Adv. Learn. Technol., pp. 89-93, 2014.

[22] B. Yousuf and O. Conlan, "VisEN: Motivating Learner Engagement Through Explorable Visual Narratives," in Design for Teaching and Learning in a Networked World, Springer, 2015, pp. 367-380.

[23] C. Coffrin, L. Corrin, P. de Barba, and G. Kennedy, "Visualizing patterns of student engagement and performance in MOOCs," Proceedins Fourth Int. Conf. Learn. Anal. Knowl. - LAK'14, pp. 83-92, 2014.

[24] R. F. Kizilcec, C. Piech, and E. Schneider, "Deconstructing Disengagement: Analyzing Learner Subpopulations in Massive Open Online Courses," Lak'13, p. 10, 2013.

[25] P. Di Bitonto, E. Pesare, V. Rossano, and T. Roselli, "Smart learning environments using social network, gamification and recommender system approaches in e- health contexts," in Smart Education and Smart e Learning, Springer International Publishing, Ed. 2015, pp. 491-500.

[26] B. Schreurs, M. de Laat, C. Teplovs, and S. Voogd, "Social Learning Analytics applied in a MOOC-environment Authors," eLearning Pap., vol. 36, no. January, pp. 45-48, 2014.

[27] R. Carrillo; E. Lavouè, and Y. Prié, "Towards Qualitative Insights for Visualizing Student Engagement in Web-based Learning Environments Rubiela Carrillo The Dimensions of Student Engagement," Proc. 25th Int. Conf. Companion World Wide Web, pp. 893-898, 2016.

[28] S. Few, Information Dashboard Design: Displaying data for at-aglance monitoring. Analytics Press, 2013.

[29] J. M. Kivikangas, G. Chanel, B. Cowley, I. Ekman, M. Salminen, S. Järvelä, and N. Ravaja, "A review of the use of psychophysiological methods in game research," J. Gaming Virtual Worlds, vol. 3, no. 3, pp. 181199, 2011.

[30] P. Bouvier, K. Sehaba, and É. Lavoué, A trace-based approach to identifying users' engagement and qualifying their engaged-behaviours in interactive systems: application to a social game. 2014.

[31] J. Lave and E. Wenger, "Situated learning: Legitimate peripheral participation," Learn. doing, vol. 95, p. 138, 1991.

[32] E. Pesare;, T. Roselli, and V. Rossano, "Engagement in social learning: Detecting engagement in online communities of practice," in International Conference on Applied Human Factors and Ergonomics, 2016, p. in press.

[33] M. Friendly and D. Denis, "The eraly origins and development of the scatterplot," J. Hist. Behav. Sci., vol. 41, no. 2, pp. 103-130, 2005.

[34] W. Aigner, S. Miksch, W. Muller, H. Schumann, and C. Tominski, "Visualizing time-oriented data-A systematic view," Comput. Graph., vol. 31, no. 3, pp. 401-409, 2007.

[35] F. Chevalier, N. H. Riche, C. Plaisant, A. Chalbi, and C. Hurter, "Animations 25 Years Later: New Roles and Opportunities," pp. 280-287, 2016.

[36] Y. Albo, J. Lanir, P. Bak, and S. Rafaeli, "Static vs . Dynamic Time Mapping in Radial Composite Indicator Visualization,” pp. 264-271, 2016.

[37] M. Tversky, B., Morrison, J. B., \& Betrancourt, "Animation : can it facilitate?,” Int. J. Hum. Comput. Stud., pp. 247-262, 2002.

[38] S. Buckingham Shum and R. Ferguson, "Social Learning Analytics," Soc. Learn. Anal., vol. 15, no. 3, pp. 3-26, 2012. 